\title{
A Condition-based Intra Prediction Algorithm for H.264/AVC
}

\author{
Jia-Wei Chen ${ }^{1}$, Chun-Hao Chang ${ }^{2}$, Chien-Chang Lin ${ }^{2}$, Yi-Huan Ou Yang ${ }^{1}$, Jiun-In Guo ${ }^{2}$, and Jinn-Shyan Wang ${ }^{1}$ \\ ${ }^{1}$ Department of Electrical Engineering, National Chung-Cheng University \\ ${ }^{2}$ Department of Computer Science and Information Engineering, National Chung-Cheng University \\ Chia-Yi, Taiwan, Republic of China \\ ${ }^{1}$ \{92jiawei, 92chusan\}@vlsi.ee.ccu.edu.tw, ${ }^{2}$ \{cch90u, 1cc90, jiguo\}@cs.ccu.edu.tw, ${ }^{1}$ ieegsw@ee.ccu.edu.tw
}

\begin{abstract}
This paper proposes a condition-based algorithm for H.264/AVC 4x4 intra prediction. Exploiting high correlation existed in neighboring intra prediction modes, we propose the three conditions to skip the less possible candidates in doing Intra4x4 block mode decision. When compared to the 9 prediction modes in the full search algorithm, the proposed algorithm can complete a $4 \mathrm{x} 4$ intra prediction using 4.4 prediction modes operation in average. The simulation result shows that the proposed algorithm can reduce computational complexity up to $44 \%$ at the cost of less than $0.1 \mathrm{~dB}$ PSNR loss in average.
\end{abstract}

\section{INTRODUCTION}

The Joint Video Team (JVT) of ISO/IEC MPEG and ITU-T VCEG has developed a new video standard, i.e. H.264/AVC, for high quality natural video coding [1]. Compared to the previous MPEG-2/H.263/MPEG-4 standards [2-4], H.264/AVC can achieve more bit-rate reduction with the same video coding quality [5]. The improvement in coding performance comes mainly from the prediction part. Among the new coding tools of H.264, the intra prediction improves the coding performance when the inter prediction fails to find a good match. It is also used to reduce the residues in the compression of still images as well.

In H.264/AVC intra frame coder, the macroblock pixel predictor is generated by neighboring reconstructed samples. Compared to JPEG-2000 using DWT 53 on still images [6], H.264 intra coding owns about $0.1 \sim 0.8 \mathrm{~dB}$ gain on PSNR. This quality gain comes from more complex intra prediction tools used in H.264/AVC than JPEG-2000. According to simulation results in [6], the functions of transform and intra predictor generation adopting full search algorithm take $77 \%$ of complexity required in H.264/AVC intra coding, which is obviously the bottleneck of the H.264 intra frame coder in the hardware implementation. Therefore, it is inevitable to develop low complexity fast algorithms for intra-prediction mode selection.

In the literatures, there have been some efficient approaches proposed on fast intra prediction search algorithms [6-9]. However, these search algorithms are more suitable for software implementation. The design [6] proposed a simple and effective method to decrease the computational complexity. The proposed method is based on the local statistics of neighboring blocks with a threshold. Due to the assumption of the local statistics of neighboring blocks is not always true, using this approach would increase the bit-rate significantly with some PSNR loss. The designs [7] and [8] proposed a new search algorithm by using a threshold to early terminate the computation of the most probable mode. When the cost of the most probable mode is smaller than the threshold, the most probable mode is selected as the best mode without any computation. Otherwise, the mode with minimum cost is selected. Using this approach needs additional executing time to determine a suitable threshold, which may complicate the control in hardware implementation. Finally, the design [9] adopted the edge pixels direction to determine the slope. Through calculating the slope, the possible mode of the intra prediction can be predicted. Similar to the designs in [7-8], the approach in design [9] is more suitable for software realization for pursuing lower complexity.

In this paper, we will propose a condition-based intra prediction algorithm for reducing the hardware complexity in H.264 intra frame coders. Based on the proposed algorithm, we can complete a $4 \times 4$ intra prediction using 4.4 operation modes in average instead of nine modes in the full search algorithm. The simulation result shows that the proposed algorithm can reduce computational complexity up to $44 \%$ at the cost of less than $0.1 \mathrm{~dB}$ PSNR loss in average.

The rest of this paper is organized as follows. In Section 2 , we will describe the proposed algorithm in more details. In Section 3, we will show the simulation results and performance comparison. Finally, we conclude this paper in Section 4.

\section{CONDITION-BASED INTRA PREDICTION ALGORITHM}

In this section, we propose a condition-based intra prediction algorithm for H.264/AVC $4 \times 4$ intra frame encoding. The proposed algorithm exploits the high data correlation existed in the current block and its neighboring $4 \times 4$ blocks in doing intra prediction. For example, if previous-encoded $4 \times 4$ blocks $\mathrm{A}$ and $\mathrm{B}$ shown in Fig. 1 were predicted using mode 2 (i.e. DC mode), it is likely that the best mode for current block $\mathrm{C}$ is also mode 2. According to above idea, we define the rules on fast deciding the best prediction mode through detecting the prediction modes of neighboring blocks. Three search algorithms are defined as follows: 1) Condition-correlation search method, 2) Halffull search method, and 3) Context-correlation search method. Each search algorithm is based on some particular conditions to search the best prediction mode. They are described in more details in the following subsections. 


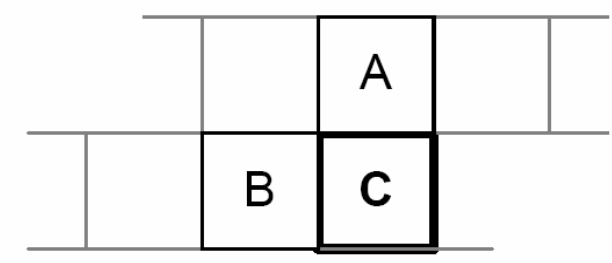

Fig. 1 Adjacent 4x4 intra coded blocks in H.264/AVC

\subsection{Condition-Correlation Search Method}

In the proposed algorithm, the neighboring block is available or not was first considered to define the initial search mode. According to the H.264 standard, only DC mode is selected if the upper block and left block are both unavailable. In addition, mode 1, 2, and 8 will be selected as candidates while the upper block is unavailable, and the mode $0,2,3$, and 7 will be selected as candidates while the left block is unavailable. The above mentioned cases usually happen either on the MB boundary conditions of intraframes or on the intra blocks of inter-frames. Otherwise, the advanced represented search mode of Intra $4 \times 4$ prediction will be selected while the neighboring blocks are all available. Thus we compose a condition-correlation search table shown in Table 1 of the above-mentioned search algorithm.

Table 1: Proposed condition-correlation search table

\begin{tabular}{|c|c|c|c|}
\hline \multicolumn{2}{|c|}{ Condition-correlation search method } \\
\cline { 3 - 4 } \multicolumn{2}{|c|}{} & Non Available & Available \\
\hline \multirow{2}{*}{$\begin{array}{c}\text { Left } \\
\text { Bloc } \\
\text { k }\end{array}$} & $\begin{array}{c}\text { Non } \\
\text { Available }\end{array}$ & $\mathbf{2}$ & $\mathbf{0 , 2 , 3 , 7}$ \\
\cline { 2 - 4 } & Available & $\mathbf{1 , 2 , 8}$ & $\begin{array}{c}\text { Half-full search or } \\
\text { Context- } \\
\text { correlation search }\end{array}$ \\
\hline
\end{tabular}

\subsection{Half-full Search Method}

Secondly, while the upper and left blocks are both available as indicated in the lower right corner of Table 1, we try to determine the best mode through the half-full search or Context-correlation search methods. Each of Intra $4 \times 4$ prediction modes has its own represented direction except the DC prediction mode. In natural images, neighboring pixels change smoothly so that the neighboring blocks own high correlation among them. Therefore, the spatial correlation of the neighboring blocks can be used to predict the possible candidates in the current block. If the DC mode is used in the neighboring blocks, all kinds of prediction modes may have possibility to be the best mode due to the fact that the DC prediction mode does not have an obvious direction. Thus we should select all the modes for candidates when the DC mode occurs in the neighboring blocks. In order to reduce the computational complexity, we select the modes in alternate direction instead of using the full search scheme. We call this approach as half-full search in the proposed algorithm. Fig. 2 shows the illustration of the half-full search method. Table 2 shows the search table used in the proposed half-full search method. As shown in Table 2, we adopt the Context-correlation search method (would be discussed later) while both the upper and left blocks are not in DC mode.

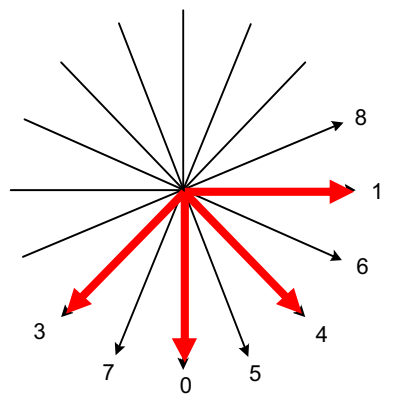

Fig. 2: Illustration of the proposed half-full search method

Table 2: Search table of the half-full search method

\begin{tabular}{|c|c|c|c|}
\hline \multicolumn{2}{|c|}{ Half-full search method } \\
\cline { 3 - 4 } \multicolumn{2}{|c|}{} & \multicolumn{2}{|c|}{ Upper Block (Available) } \\
\hline \multirow{2}{*}{$\begin{array}{c}\text { Left Block } \\
\text { (Available) }\end{array}$} & $\begin{array}{c}\text { DC } \\
\text { The other } \\
\text { Prediction } \\
\text { Modes }\end{array}$ & $\mathbf{0 , 1 , 2 , 3 , 4}$ & $\mathbf{0 , 1 , 2 , 3 , 4}$ \\
& $\begin{array}{c}\text { P, 1, 2, 3, 4 } \\
\text { Context- } \\
\text { correlation Search }\end{array}$ \\
\hline
\end{tabular}

\subsection{Context-Correlation Search Method}

Besides the DC prediction mode, the other prediction modes have their own directional characteristics as we mentioned above. After exploiting the above mentioned two search methods, we further consider the smooth variation of real images and assume that the prediction modes between neighboring blocks will not change too much in determining the best mode in the cases indicated in lower right corner of Table 2. We propose a context-correlation search method for this purpose. That is, in addition to the two prediction modes respectively for the top and left blocks, we also select the modes with the approximate directions to those of the top and left block as the mode decision candidates. We take a simple example shown in Fig. 4 for illustrating the proposed context-correlation search method. The prediction modes of the top and left blocks are given as mode 6 and mode 7, respectively. According to the above search method, we will select the modes 3, 7, 0 (from the prediction mode 7), the modes $4,6,1$ (from the prediction mode 6), and mode 2 (i.e. the DC prediction mode) as the candidates for best prediction mode. 


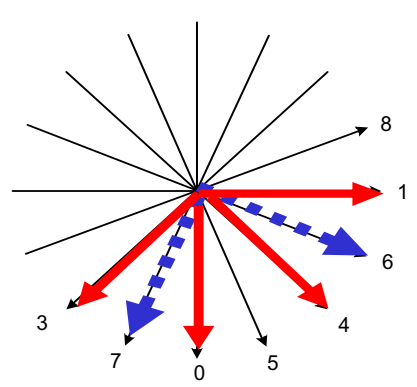

Fig. 4: An example for the proposed context-correlation search method

According to the above mentioned three search methods, we can make decision on the Intra $4 \times 4$ best prediction mode for all the cases shown in Table 3 (which is located at the bottom of page 4). Here is some analysis on the characteristics of the proposed search algorithm. In the condition-correlation search method shown in Table 1, we can make sure that we have considered all the condition cases and employed the properties of unavailable blocks. Regarding the available neighboring block cases, two methods were proposed to further skip the candidates. By exploiting the properties of real images and spatial correlations, the improbable prediction modes were eliminated to reduce the complexity. The proposed half-full search method can effectively reduce the computational complexity, but it suffers from error prediction. This is because we do not search all the prediction modes as the full-search algorithm in H.264 reference software (JM). Nevertheless, the proposed context-correlation search method will solve the error propagation problem. Even if an error exists in the prediction mode of the neighboring blocks, the context-correlation search will also select the approximate modes to be the candidates. Therefore, adopting the context-correlation search method can choose the modes which are close to the best ones provided that the coded frame will not change abruptly.

\section{SIMULATION RESULTS AND PERFORMANCE COMPARISON}

In this section, we will show the simulation results of the proposed algorithm as compared to the search algorithm proposed in [6] on three different CIF videos, including Foreman, Mobile, and Stefan. For each video, we encode them in 300 frames with H.264 intra frame coder with SATD mode decision. At the same time, we turn off the function of the rate distortion optimization (RDO). In order to measure the computational complexity, we simulate the video with $300 \mathrm{I}$-frames under several different fixed QP values. Tables 4,5 , and 6 respectively show the quality drop and complexity reduction when using JM reference software, the proposed algorithm, and the existing algorithm presented in [6]. In the tables, we can find the proposed algorithm can reduce the computational complexity up to $44 \%$ at the cost of less than $0.07 \mathrm{~dB}$ loss in PSNR, as compared to the full search algorithm adopted in JM.
Besides, the proposed algorithm can reduce the complexity up to $14 \%$ and even has better quality than the existing algorithm presented in [6].

Besides, in order to evaluate the quality of the proposed algorithm on the H.264 intra frame coder, we use ratedistortion curve. We compare the performance of the proposed algorithm, the search algorithm presented in [6], and the full search algorithm used in JM. Figures 5, 6 and 7 respectively show the rate-distortion curves of the above mentioned three algorithms using Foreman, Mobile, and Stefan sequences. We can find that the performance of the proposed intra frame coder is better than that using the algorithm in [6] at the same bit-rate. In summary, through simulation result shown in Figures 5 7, we can conclude that the proposed algorithm outperforms the algorithm in [6] in terms of less complexity and better quality.

\section{CONCLUSION}

In this paper we have proposed a new condition-based intra prediction algorithm for H.264/AVC. It can reduce the $44 \%$ of computational complexity at the cost of only $0.02 \%$ PSNR loss as compared to JM. Moreover, the proposed algorithm can reduce the complexity up to $14 \%$ and even has better quality than the existing algorithm presented in [6].

Table 4: Comparison of search algorithm in $\mathrm{Qp}=25$

\begin{tabular}{|c|c|c|c|c|}
\hline \multicolumn{2}{|c|}{ Sequences } & Foreman & Mobile & Stefan \\
\hline $\begin{array}{l}\text { JM } \\
\text { (a1) }\end{array}$ & $\begin{array}{c}\text { PSNR } \\
\text { (Calculation) }\end{array}$ & $\begin{array}{c}39.42 \\
(16841700)\end{array}$ & $\begin{array}{c}37.79 \\
(16841700)\end{array}$ & $\begin{array}{c}38.99 \\
(16841700)\end{array}$ \\
\hline $\begin{array}{l}\text { Huang's [6] } \\
\text { (a2) }\end{array}$ & $\begin{array}{c}\text { PSNR } \\
\text { (Calculation) }\end{array}$ & $\begin{array}{c}39.36 \\
(10909075)\end{array}$ & $\begin{array}{c}37.76 \\
(11481180)\end{array}$ & $\begin{array}{c}38.97 \\
(10954995)\end{array}$ \\
\hline $\begin{array}{l}\text { Proposed } \\
\text { (a3) }\end{array}$ & $\begin{array}{c}\text { PSNR } \\
\text { (Calculation) } \\
\end{array}$ & $\begin{array}{c}39.39 \\
(9425977) \\
\end{array}$ & $\begin{array}{c}37.78 \\
(9575309)\end{array}$ & $\begin{array}{c}38.98 \\
(9299478)\end{array}$ \\
\hline \multirow{2}{*}{$\begin{array}{c}\text { Comparison } \\
\text { PSNR }\end{array}$} & $(\mathrm{a} 3-\mathrm{a} 1)$ & -0.03 & -0.01 & -0.01 \\
\hline & $(\mathrm{a} 3-\mathrm{a} 2)$ & 0.03 & 0.02 & 0.01 \\
\hline \multirow{2}{*}{$\begin{array}{l}\text { Comparison } \\
\text { Calculation }\end{array}$} & $(\mathrm{a} 1-\mathrm{a} 3) / \mathrm{a} 1$ & $44 \%$ & $43 \%$ & $45 \%$ \\
\hline & $(\mathrm{a} 2-\mathrm{a} 3) / \mathrm{a} 2$ & $14 \%$ & $17 \%$ & $15 \%$ \\
\hline
\end{tabular}

Table 5: Comparison of search algorithm in $\mathrm{Qp}=30$

\begin{tabular}{|c|c|c|c|c|}
\hline \multicolumn{2}{|c|}{ Sequences } & Foreman & Mobile & Stefan \\
\hline $\begin{array}{l}\text { JM } \\
\text { (a1) }\end{array}$ & $\begin{array}{c}\text { PSNR } \\
\text { (Calculation) }\end{array}$ & $\begin{array}{c}35.91 \\
(16841700) \\
\end{array}$ & $\begin{array}{c}33.18 \\
(16841700)\end{array}$ & $\begin{array}{c}34.65 \\
(16841700)\end{array}$ \\
\hline $\begin{array}{l}\text { Huang's [6] } \\
\text { (a2) }\end{array}$ & $\begin{array}{c}\text { PSNR } \\
\text { (Calculation) }\end{array}$ & $\begin{array}{c}35.82 \\
(10861120)\end{array}$ & $\begin{array}{c}33.14 \\
(11411965)\end{array}$ & $\begin{array}{c}34.61 \\
(10902760)\end{array}$ \\
\hline $\begin{array}{l}\text { Proposed } \\
\text { (a3) }\end{array}$ & $\begin{array}{c}\text { PSNR } \\
\text { (Calculation) }\end{array}$ & $\begin{array}{c}35.86 \\
(9330572) \\
\end{array}$ & $\begin{array}{c}33.16 \\
(9514270)\end{array}$ & $\begin{array}{c}34.64 \\
(9305862)\end{array}$ \\
\hline \multirow{2}{*}{$\begin{array}{l}\text { Comparison } \\
\text { PSNR }\end{array}$} & $(a 3-a 1)$ & -0.05 & -0.02 & -0.01 \\
\hline & $(\mathrm{a} 3-\mathrm{a} 2)$ & 0.04 & 0.02 & 0.03 \\
\hline \multirow{2}{*}{$\begin{array}{l}\text { Comparison } \\
\text { Calculation }\end{array}$} & $(\mathrm{a} 1-\mathrm{a} 3) / \mathrm{a} 1$ & $44 \%$ & $43 \%$ & $45 \%$ \\
\hline & $(a 2-a 3) / a 2$ & $14 \%$ & $17 \%$ & $15 \%$ \\
\hline
\end{tabular}


Table 6: Comparison of search algorithm in $\mathrm{Qp}=35$

\begin{tabular}{|c|c|c|c|c|}
\hline \multicolumn{2}{|c|}{ Sequences } & Foreman & Mobile & Stefan \\
\hline $\begin{array}{l}\text { JM } \\
\text { (a1) }\end{array}$ & $\begin{array}{c}\text { PSNR } \\
\text { (Calculation) } \\
\end{array}$ & $\begin{array}{c}32.8 \\
(16841700) \\
\end{array}$ & $\begin{array}{c}29.03 \\
(16841700)\end{array}$ & $\begin{array}{c}30.68 \\
(16841700)\end{array}$ \\
\hline $\begin{array}{l}\text { Huang's [6] } \\
\text { (a2) }\end{array}$ & $\begin{array}{c}\text { PSNR } \\
\text { (Calculation) }\end{array}$ & $\begin{array}{c}32.69 \\
(10841340)\end{array}$ & $\begin{array}{c}28.95 \\
(11331375)\end{array}$ & $\begin{array}{c}30.61 \\
(10836335)\end{array}$ \\
\hline $\begin{array}{l}\text { Proposed } \\
\text { (a3) }\end{array}$ & $\begin{array}{c}\text { PSNR } \\
\text { (Calculation) }\end{array}$ & $\begin{array}{c}32.73 \\
(9278633) \\
\end{array}$ & $\begin{array}{c}28.99 \\
(9458454)\end{array}$ & $\begin{array}{c}30.64 \\
(9320992)\end{array}$ \\
\hline \multirow{2}{*}{$\begin{array}{c}\text { Comparison } \\
\text { PSNR }\end{array}$} & $(\mathrm{a} 3-\mathrm{a} 1)$ & -0.07 & -0.04 & -0.04 \\
\hline & $(\mathrm{a} 3-\mathrm{a} 2)$ & 0.04 & 0.04 & 0.03 \\
\hline \multirow{2}{*}{$\begin{array}{l}\text { Comparison } \\
\text { Calculation }\end{array}$} & $(\mathrm{a} 1-\mathrm{a} 3) / \mathrm{a} 1$ & $45 \%$ & $44 \%$ & $45 \%$ \\
\hline & $(a 2-a 3) / a 2$ & $14 \%$ & $17 \%$ & $14 \%$ \\
\hline
\end{tabular}

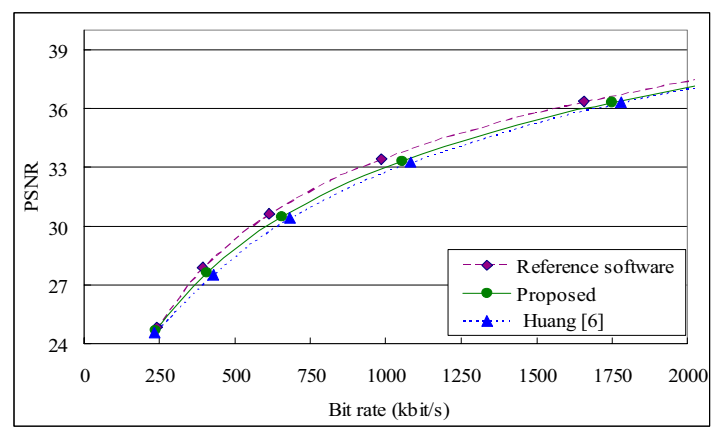

Fig. 5: Rate-distortion curves of the proposed intra coding algorithm using Foreman sequence

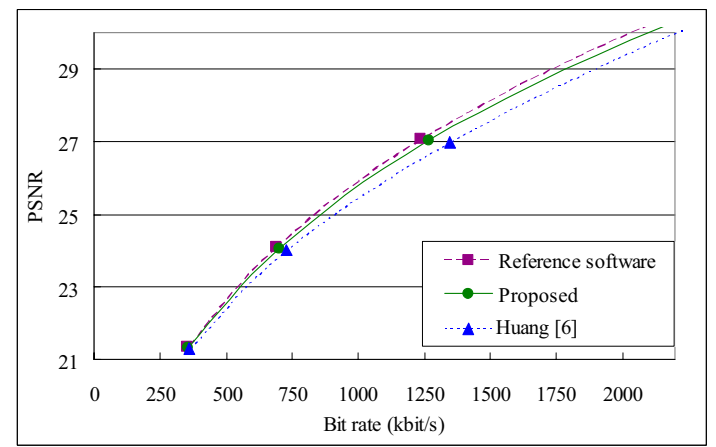

Fig. 6: Rate-distortion curves of the proposed intra coding algorithm using Mobile sequence

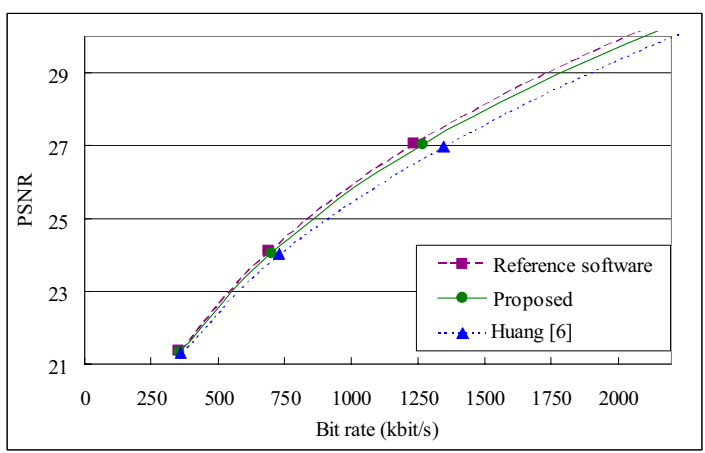

Fig. 7: Rate-distortion curves of the proposed intra coding algorithm using Stefan sequence

\section{References}

[1] Joint Video Team, Draft ITU-T Recommendation and Final Draft International Standard of Joint Video Specification, ITU-T Recom. H.264 and ISO/IEC 14496-10 AVC, May 2003.

[2] "Generic Coding of Moving Pictures and Associated Audio Information - Part 2: Video," ITU-T and ISO/IEC JTC1, ITU-T Recommendation H.262-ISO/IEC 138 18-2 (MPEG-2), 1994.

[3] "Video Coding for Low Bitrate Communication Version 1," ITU-T, ITU-T Recommendation H.263, 1995.

[4] "Coding of Audio-Visual Objects-Part 2: Visual," ISO/IEC JTC1, ISO/IEC 14 496-2 (MPEG-4 visual version 1), 1999.

[5] A. Joch, F. Kossentini, H. Schwarz, T. Wiegand, and G. J. Sullivan," Performance comparison of video coding standards using Lagragian coder control,“ Proc. ICIP2002, pp. 501-504.

[6] Y. W. Huang; B. Y. Hsieh; T. C. Chen; L. G. Chen; "Analysis, fast algorithm, and VLSI architecture design for H.264/AVC intra frame coder," IEEE Transactions on Circuits and Systems for Video Technology, pp. 378-401, March 2005.

[7] Meng, B.; Au, O.C, "Fast intra-prediction mode selection for $4 \times 4$ blocks in H.264," Proc. ICASSP2003, pp.III 389-92, April 2003.

[8] Meng, B.; Au, O.C, Chi-Wah Wong, Hong-Kwai Lam, "Efficient intraprediction mode selection for $4 \times 4$ blocks in H.264," Proc. ICME2003, pp.III 521-4, July 2003.

[9] F. Pan, X. Lin, R. Susanto, K. P. Lim, Z. G. Li, G. N. Feng, D. J. Wu, and S. Wu, "Fast Mode Decision for Intra Prediction in H.264/AVC Video Coding," JVT-G013, $7^{\text {th }}$ Meeting, Pattaya II, Thailand, 7-14 March, 2003.

Table 3: Search table for the mode decision in the proposed condition-based intra prediction algorithm

\begin{tabular}{|c|c|c|c||c|c|c|c|c|c|c|}
\hline $\mathbf{B} \backslash \mathbf{A}$ & unavailable & $\mathbf{0}$ & $\mathbf{1}$ & $\mathbf{2}$ & $\mathbf{3}$ & $\mathbf{4}$ & $\mathbf{5}$ & $\mathbf{6}$ & $\mathbf{7}$ & $\mathbf{8}$ \\
\hline unavailable & 2 & 2037 & 2037 & 2037 & 2037 & 2037 & 2037 & 2037 & 2037 & 2037 \\
\hline $\mathbf{0}$ & 281 & 2057 & 2057168 & 01234 & 23705 & 205746 & 20574 & 2057614 & 20573 & 205781 \\
\hline $\mathbf{1}$ & 281 & 2057168 & 2168 & 01234 & 237168 & 246518 & 2540168 & 26148 & 2703168 & 2816 \\
\hline $\mathbf{2}$ & 281 & 01234 & 01234 & 01234 & 01234 & 01234 & 01234 & 01234 & 01234 & 01234 \\
\hline $\mathbf{3}$ & 281 & 20573 & 237168 & 01234 & 237 & 246537 & 254037 & 261437 & 2703 & 28137 \\
\hline $\mathbf{4}$ & 281 & 205746 & 216845 & 01234 & 237465 & 2465 & 25406 & 26145 & 2703465 & 281465 \\
\hline $\mathbf{5}$ & 281 & 20574 & 2168540 & 01234 & 237540 & 24650 & 2540 & 261450 & 270354 & 281540 \\
\hline $\mathbf{6}$ & 281 & 2057614 & 21684 & 01234 & 237614 & 24651 & 254061 & 2614 & 2703614 & 28164 \\
\hline $\mathbf{7}$ & 281 & 20573 & 2168703 & 01234 & 2370 & 2465703 & 254073 & 2614703 & 2703 & 281703 \\
\hline $\mathbf{8}$ & 281 & 205781 & 2168 & 01234 & 23781 & 246581 & 254081 & 26148 & 270381 & 281 \\
\hline
\end{tabular}

